\title{
LA SEMAFORIZACIÓN NUTRICIONAL Y SU INFLUENCIA EN LA SALUD DE LA POBLACIÓN ECUATORIANA
}

\section{The Reflections on nutritional frog and its influence health of ecuatoriana population}

\section{Argenis Montilla Pacheco ${ }^{a}$, José Vicente Becerra ${ }^{b}$ y Luis Reinaldo Reyes Chávez}

Recibido: 6 de junio, 2019 • Aprobado: 2 de septiembre, 2019

Cómo citar: Montilla Pacheco A, Vicente Becerra J, Reyes Chávez LR. La semaforización nutricional y su influencia en la salud de la población ecuatoriana. cysa [Internet]. 25 de noviembre de 2019 [citado 28 de noviembre de 2019];3(3):15-2. Disponible en: https:// revistas.intec.edu.do/index.php/cisa/article/view/1544

Resumen

Las naciones desarrolladas propician ejemplos para demostrar que la gobernabilidad depende de la atención a factores específicos relacionados con la educación, nutrición y salud. Por su parte, los países que aspiran alcanzar mayores niveles de desarrollo, entre ellos Ecuador, han considerado una serie de acciones en salud que demuestran preocupación por el bienestar de sus ciudadanos; una de ellas es la implementación de la semaforización nutricional, cuyo propósito es informar al consumidor sobre los nutrientes por ración que contienen las bebidas y alimentos ya elaborados. En ese sentido, el objetivo de este trabajo es analizar las ventajas e impactos que esta medida puede tener sobre la salud de la población ecuatoriana. El trabajo es un ensayo construido a partir de la hermenéutica, basada en la revisión, análisis e interpretación de distintas fuentes documentales que abordan el tema alimentario. Se concluye que la semaforización nutricional es de importancia para la salud de la población, y que esta debe abarcar no solamente los alimentos y bebidas elaborados industrialmente, sino también aquellos que se preparan en diferentes expendios de comida, especialmente en restaurantes.

Palabras clave: alimentación; diabetes, hipertensión, nutrición; salud.

\footnotetext{
a Docente de la Universidad Laica Eloy Alfaro de Manabí Editor Interno de la Revista Electrónica REFCAIE

Correo-e: argenismontilla@hotmail.com
}

\begin{abstract}
Developed nations propitiate examples to demonstrate that governance depends on attention to specific factors related to education nutrition and health. For their part, countries that aspire to reach important higher levels of development, including Ecuador, has considered a series of health actions that show concern for the welfare of their citizens; one of them is the implementation of the nutritional frog, the purpose of which is to inform the consumer of the nutrients per ration containing the beverages and processed foods. In this sense, the objective of this work is to analyze the advantages and impacts that this measure can have on the health of the ecuadorian population. The work is an essay constructed from hermeneutics, based on the revision of different documentary sources that address the food theme. It Is concluded that the nutritional frog is of importance for the health of the population, and that this must include not only the food and beverages elaborated industrially, but also, those that are prepared in different food outlets, Especially in restaurants.
\end{abstract}

Keywords: Feeding; diabetes, hypertension, nutrition, health.

\footnotetext{
b Universidad Laica Eloy Alfaro de Manabí orcid.org/0000-0002-9466-0645

Correo-e: becerrajo@gmail.com

${ }^{\mathrm{c}}$ Universidad Laica Eloy Alfaro de Manabí orcid.org/0000-0002-0855-9904

Correo-e: luis.reyes@uleam.edu.ec
} 


\section{Introducción}

La salud es el estado en el cual la persona disfruta del bienestar integral, es decir, físico, mental y social; esta definición quedó anclada desde 1948 por la Organización Mundial de la Salud ${ }^{1}$. De allí que una sana alimentación vendría a ser el soporte sobre el que se cimenta ese bienestar, específicamente cuando se satisfacen plenamente las necesidades nutricionales de las personas.

Los hábitos alimenticios generados por las propias costumbres y estilos de vida de la población, junto a la creación de necesidades artificiales y las tendencias globales del mercado, han propiciado la producción y venta a gran escala de diversos productos alimenticios poco saludables, que se expresan como parte de las causas que conducen a la población a carecer de una salud plena, que padece y sufre por el incremento de enfermedades de distintos tipos, entre ellas, obesidad, diabetes e hipertensión.

Ecuador era uno de los países de América con menor prevalencia de obesidad para el 2010, no obstante, para el año 2013, 6 de cada 10 personas entre 19 y 60 años presentaban sobrepeso y obesidad, lo cual representa el $60 \%$ de la población que compone ese grupo etario $^{2}$. De igual manera, las estadísticas demuestran la prevalencia de diabetes mellitus en la nación (11\% de las personas mayores de 50 años en el año 2016), de modo que este mal se ha convertido en un serio problema de salud pública, tanto para el gobierno central como para los gobiernos provinciales.

"El surgimiento de la obesidad, además de la hipertensión arterial y patologías cardiacas y cerebrovasculares conducen al aumento de la tasa de mortalidad de los ecuatorianos" 3 , pues en un contexto más amplio, de acuerdo con Medina, Aguilar y Solé-Sedeño "es el resultado, entre otros factores, de la ignorancia que en materia alimenticia persiste en buena parte de la población"’.
De igual manera, el sobrepeso y la obesidad en los escolares está en el orden del 29,9\% y en adolescentes $26 \%$, mientras que en adultos es de $62,8 \%$. Estas patologías se cuentan entre las principales causas de muerte en el país en el año $2013^{5}$, que en conjunto, produjeron el fallecimiento de 15.393 personas, es decir, 42 ecuatorianos por día (gráfica 1).

Gráfica 1. Causas de defunción en el Ecuador para el año 2011

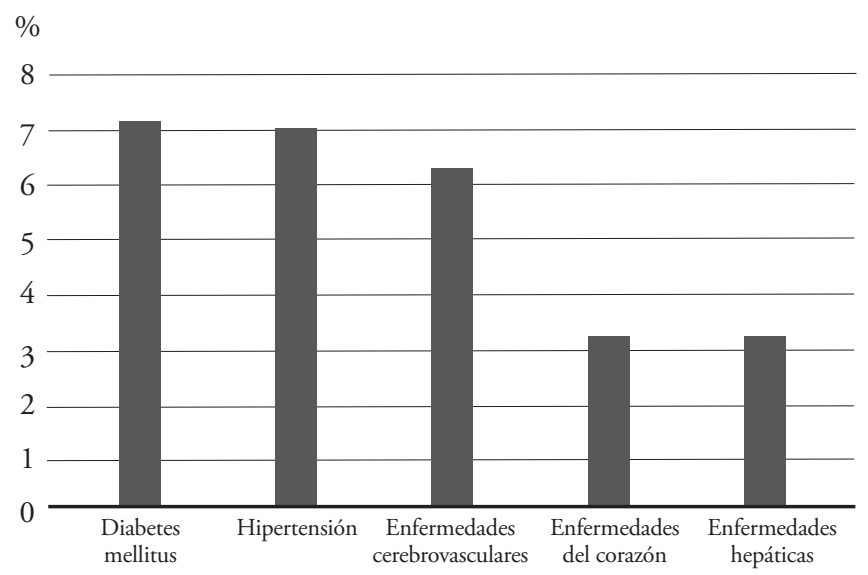

Fuente: elaboración propia a partir de datos del Instituto Nacional de Estadísticas y Censos 5 .

Por otra parte, la ignorancia de las personas sobre la importancia de una alimentación sana está influyendo negativamente en la salud de la población, con consecuencias económicas que afectan los presupuestos estatales dirigidos a la atención de salud gratuita; de forma que a menor conocimiento menor salud, pues el cuerpo es una construcción simbólica, es el resultado de una construcción social y cultural ${ }^{6}$, de allí que se puede entender que la buena alimentación no es un conocimiento intrínseco en los individuos, más bien se adquiere a través de la educación y la concienciación, accediendo a alimentos sanos, y desde luego, valorando la salud y la vida.

Anteriormente, un paseo por anaqueles de abastos y supermercados en distintas ciudades de Ecuador era suficiente para determinar la gran variedad de productos que en estos se expendían. Muchos de ellos 
sin ningún valor nutricional, pero promocionados por fuertes campañas de marketing que impactaban directamente sobre el consumidor, de manera que los mismos terminaban en las despensas de los hogares, generando consecuencias que se manifiestan en el crecimiento exponencial de diversas enfermedades asociadas a malos hábitos alimenticios, afectando la salud y la calidad de vida de los consumidores.

Debido al problema descrito, y estimulado por el éxito de la activación de esta estrategia en el Reino Unido, el Estado ecuatoriano implementó en el año 2013 un sistema de semaforización de alimentos ${ }^{7}$, con la intención de que el consumidor sea más consciente a la hora de seleccionarlos, valorando su importancia en materia de salud. Sin embargo, esta acción de protección no ha tenido el impacto esperado, y tampoco ha llegado a extenderse a los alimentos que se expenden preparados en restaurantes y demás establecimientos de comidas como para afirmar que hay una amplia cobertura que garantice una población más sana y productiva, que redunde en una importante disminución de gastos al Estado, concretamente en materia de salud.

Finalmente, por la relevancia que implica el tema de salud y alimentación, este trabajo, además del análisis del caso, busca llamar la atención al Ministerio de Salud Ecuatoriano, en el sentido de ampliar la cobertura del control de semaforización en alimentos preparados, pues es notoria la existencia de un creciente flujo de consumidores, entre ellos los turistas.

Parte de la información que acá se presenta es recogida de un proyecto de investigación que se intitula "Semaforización nutricional de los productos gastronómicos que se expenden en la Playa Murciélago de la ciudad de Manta" ejecutados por la Facultad de Hospitalidad, Hotelería y Turismo de la Universidad Laica Eloy Alfaro de Manabí, Ecuador. Este proyecto se planteó entre sus objetivos, determinar el contenido nutricional y calórico de los alimentos que se expenden en restaurantes del balneario, capacitar al personal de los restaurantes para contribuir con la preparación de alimentos sanos en busca de buena salud e implementar la semaforización nutricional en los productos gastronómicos que se expenden en la mencionada playa.

\section{Los contenidos normativos del derecho a la ali- mentación}

"El Comité de Derechos Económicos, Sociales y Culturales (CDESC) de la Organización de Naciones Unidas (ONU) fue el ente responsable de la elaboración de los contenidos normativos del derecho a la alimentación plasmados en la norma interpretativa conocida como Observación General No. 12" (8 p121).

Estos mismos autores agregan que esta surgió como seguimiento a la Cumbre Mundial sobre la Alimentación realizada en el 2002 y su Plan de Acción, en donde se tanteó que el derecho a la alimentación adecuada se ejerce únicamente cuando la persona, independientemente de su edad, tiene acceso físico y económico en todo momento a la alimentación adecuada, sana y nutritiva.

\section{Antecedentes del sistema de semaforización nutri- cional en Ecuador}

A partir del 18 de noviembre de 2013 las dependencias del Ejecutivo Nacional, como el Ministerio Coordinador de Producción, Empleo y Competitividad (MCPEC), de Industrias y Productividad (MIPRO), Coordinador de Desarrollo Social (MCDS) y la Agencia Nacional de Regulación, Control y Vigilancia Sanitaria (ARCSA), expidieron el Reglamento Sanitario para el Etiquetado de Alimentos Procesados de Consumo Humano con el objeto de regular, controlar y garantizar el derecho constitucional de los ciudadanos a la información oportuna, clara, precisa y honesta sobre el contenido y características de los alimentos que consumen los ecuatorianos?. 
"El reglamento determina que las industrias deben publicar los niveles de grasa, azúcar y sal que contienen los productos" ", situación que originó un cambió en el uso de ingredientes en busca de la alimentación saludable, basados en la pirámide alimentaria nutricional que promueve la Organización Mundial de la Salud (OMS), la cual, de manera gráfica, representa las porciones y la frecuencia con la que hay que consumir cada tipo o grupo de alimentos. Como su nombre lo indica, es una pirámide con pisos que se van afilando hasta la cumbre o punta ${ }^{10}$, pero que además indica la variedad de alimentos que se debe ingerir en forma habitual según los niveles, es decir, cereales y tubérculos (40\%); verduras y hortalizas (30\%); leguminosas y alimentos de origen animal (20\%); y por último, grasas y azúcares $(10 \%)$.

\section{Acerca de la semaforización de alimentos}

Arizaga, estima que:

"la información es un aspecto esencial, pero no es cuestión que la etiqueta indique que el producto contiene exceso de un componente o de otro, sino que la gente sepa por qué es necesario o por qué hay que abstenerse de algo" ${ }^{11}$.

En tal sentido, es comprensible que actualmente algunas empresas, en atención a la normativa alimentaria vigente, se presenten renuentes al establecimiento de la semaforización, ya que al colocar una etiqueta que indique la cantidad de grasa, azúcar y sal en sus productos, provocaría cierto rechazo en las personas de manera habitual los compran; al ver las señalizaciones pensarán mejor antes de comprarlos o consumirlos, impactando negativamente en las ventas de sus productos. No obstante, algunos autores ${ }^{12}$ mencionan que las personas no cambian sus hábitos alimentarios fácilmente, por cuanto la población no percibe a priori los riesgos de sus hábitos inadecuados para su salud y los más jóvenes creen que las consecuencias de comer mal les pasan a otros, o bien, se contemplan a muy largo plazo.
De acuerdo con esto, la Universidad Laica Eloy Alfaro de Manabí ${ }^{13}$, señala que la implementación del sistema semáforo requiere de un consenso entre la industria y los gobiernos y una educación nutricional ciudadana donde se valore la vida, sobre la base de los alimentos que se consumen; por tanto, la OMS recomienda a los Estados implementar acciones que corrijan los malos hábitos alimenticios y se apliquen mecanismos que permitan acceder y consumir alimentos sanos.

Para De Luis Román ${ }^{14}$, la semaforización de alimentos o etiquetado nutricional se ve como una descripción de la relación con el aporte de energía y nutrientes, a diferencia del listado de ingredientes, que es obligatorio únicamente cuando el etiquetado hace declaraciones nutricionales o propiedades saludables del producto.

Hoyos, Yance y Rendón. indican que "la semaforización debe contener la descripción de concentraciones a través de los colores rojo, que es alto; amarillo que indica el contenido medio, y verde expresando el bajo contenido" ${ }^{15}$. En específico, cada color del semáforo nutricional significa lo siguiente:

- Verde: alimentos con bajo contenido calórico, que se pueden consumir a diario, a libre demanda, son bajos en azúcar y grasa, son ricos en fibra, vitaminas, minerales y antioxidantes.

- Amarillo: alimentos con mediano contenido calórico, que se pueden consumir diariamente pero no a libre demanda, moderadamente y tomando en cuenta la porción adecuada para cada persona; $y$

- Rojo: alimentos con alto contenido calórico, cuyo consumo excesivo pueden ser nocivos para nuestro cuerpo, pudiendo ocasionar sobrepeso, obesidad, y enfermedades cardiovasculares, entre otras.

A fin de cuentas, esta medida lo que procura es que los consumidores puedan tener mejores referencias 
al momento de adquirir aquellos productos que contribuyan en el mantenimiento de su salud.

Además de lo anterior, para implementar el sistema de semaforización de alimentos en Ecuador, hay que considerar lo que establece la Norma Técnica Ecuatoriana (NTE INEN) 1334-3:2011, que señala los requisitos mínimos que deben cumplir los rótulos o etiquetas en los envases o empaques en que se expenden los productos alimenticios para consumo humano, en los que se haga de manera voluntaria, declaraciones de propiedades nutricionales y saludables.

La Norma Técnica Ecuatoriana se debe aplicar a todo producto alimenticio procesado, envasado y empaquetado que se ofrezca como tal para la venta directa al consumidor y para fines de hostelería, en los cuales se observen declaraciones de propiedades nutricionales y saludables ${ }^{16}$. De tal manera, se entiende así a cualquier representación que afirme, sugiera o implique que un alimento posee propiedades nutritivas particulares, incluyendo, pero no limitándose a su valor energético y contenido de proteínas, grasas y carbohidratos; de igual modo, a su contenido de vitaminas y minerales, y no constituyendo declaraciones de propiedades nutricionales las siguientes: mención de sustancias en la lista de ingredientes; mención de nutrientes como parte obligatoria del etiquetado nutricional; declaración cuantitativa o cualitativa de ciertos nutrientes $o$ ingredientes en la etiqueta.

\section{Impactos de la implementación de la semafori- zación de alimentos en la salud de la población ecuatoriana}

La implementación de un sistema de semaforización debe apuntar, por una parte, hacia la generación de impactos positivos de distinta naturaleza, pero especialmente a crear una cultura nutricional correcta $y$, en consecuencia, a mejorar la calidad de vida de la población. De la implantación de un óptimo sistema de semaforización nutricional se espera, además de lo anterior, un cambio de aptitud en los consu- midores expresado en buenos hábitos alimenticios, así como una mejora sustancial en la calidad de los productos alimenticios que se expenden.

La semaforización nutricional en Ecuador entró en vigor en el año 2014, fecha en la que se esperaba que su implementación propiciara cambios en materia de salud. Sin embargo, revisando los datos, el Instituto Nacional de Estadística y Censo ${ }^{5}$, reporta que las enfermedades isquémicas del corazón continúan desde 2014 con un incremento progresivo, hasta presentarse por encima de tres puntos porcentuales en el año 2016 con respecto 2014, tal como se observa en la gráfica 2.

Gráfica 2. Tasas de mortalidad por enfermedades isquémicas del corazón en la población de Ecuador 2001-2016

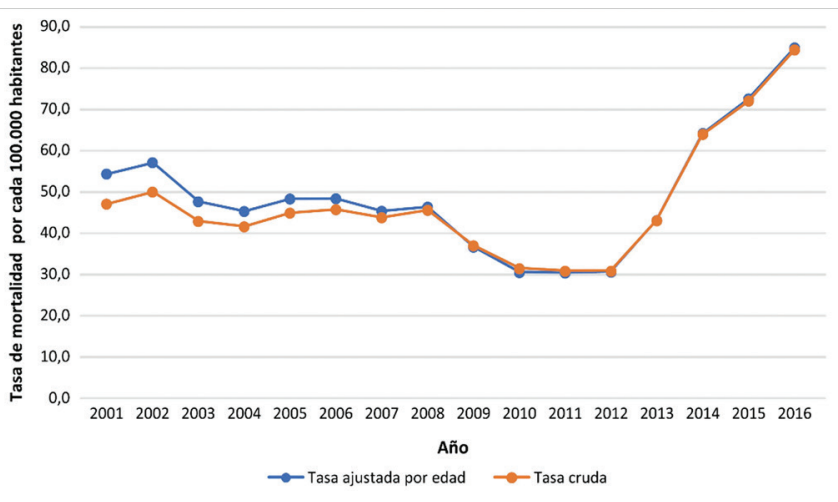

Fuente: Núñez, Aulestiaz, Borja y Simancas ${ }^{17}$.

De igual manera ocurre con la diabetes mellitus, pues se reporta como la segunda causa de muerte desde 2014, año a partir del cual mantiene esa posición, incluso con un repunte de alrededor 27 décimas en los años siguientes. Moreno et al., ${ }^{18}$ señalan que exponerse a déficits nutricionales a temprana edad provoca fisiopatológicamente cambios endoteliales, mayor actividad del sistema renina-angiotesina $\mathrm{y}$ alteraciones en el metabolismo que predisponen precozmente a hipertensión y diabetes, sumado a la poca adherencia al tratamiento una vez que se presenta. 
"La enfermedad que ocupa el tercer lugar como causa de fallecimientos en el país es de tipo cerebrovascular, tienen un repunte de 34 décimas porcentuales en 2015 con respecto al 2014 y se mantienen constantes hasta el presente" ${ }^{17}$. Esta patología desde 1990 se convirtió en la primera causa de mortalidad en Ecuador y se transforma en una amenaza para el sistema de salud, ya que el $85 \%$ de los cuidados reciben ese tipo de atención en instituciones públicas ${ }^{17}$.

Finalmente, se encuentran las enfermedades hipertensivas que, a diferencia de las anteriores, son desplazadas al quinto lugar en el año 2017 por la influenza y neumonía, de modo que muestran un decrecimiento de 77 décimas en el intervalo correspondiente entre 2014 y 2017. No obstante, en Ecuador existen pocos trabajos de investigación dedicados al estudio de estas patologías a pesar de los altos riesgos que implican para su población, sin embargo, los que existen concluyen que las enfermedades que hoy son las principales causas de muerte en el país siguen siendo las mismas desde hace más de 25 ańos.

Sobre el particular, algunos autores ${ }^{18}$ expresan que a partir de 2012 se han implementado estrategias enfocadas en contrarrestar el efecto de los principales factores de riesgo cardiovascular, como la campaña "Ecuador libre de Humo de Tabaco" y el programa "Ecuador ejercítate y vive sano" y el sistema de semaforización nutricional de alimentos en el 2013, pero ninguna de estas estrategias ha sido evaluada en profundidad como para estimar su impacto en la población.

\section{Conclusiones}

La estrategia de semaforización nutricional implementada en la República del Ecuador no ha sido suficiente para disminuir de manera significativa las enfermedades asociadas a malos hábitos alimentarios. A la vez, se aprecia como un sistema simple, pues el etiquetado se limita a señalar los niveles de concentración de únicamente tres elementos, grasa, azúcar y sal.

En forma general, los índices de enfermedades asociadas a la mala alimentación no acusan una disminución significativa, por el contrario, han aumentado después del año 2014, que es cuando entra en vigor el sistema de semaforización nutricional.

Se puede señalar sobre la base de las estadísticas encontradas que los resultados en materia de salud no han sido alcanzados en las dimensiones esperadas, de allí que pareciera oportuno sugerir la implantación de un proyecto nacional de prevención de enfermedades que abarque estrategias desde la educación formal e informal, a través de campañas donde se sumen todas las instituciones del Estado, haciendo seguimiento permanente a los pacientes de consulta externa y evaluando programas implementados, tales como el de semaforización, que aunque es positivo, por sí solo no ha contribuido a mejorar los resultados para los que fue creado.

Se concluye que la semaforización nutricional es de importancia para la salud de la población, y que esta debe abarcar no solamente los alimentos y bebidas elaborados industrialmente, sino también aquellos que se preparan en diferentes expendios de comida, especialmente en restaurantes.

\section{Agradecimientos}

Este trabajo es parte de los resultados del proyecto de investigación: Semaforización nutricional de alimentos que se expenden en restaurantes de la playa $E l$ Murciélago, Manta, provincia de Manabi, Ecuador. Financiado por la Universidad Laica Eloy Alfaro de Manabí. Agradecemos la colaboración de los estudiantes de la carrera de Hotelería, así como del personal que conforma la planta de trabajadores de los restaurantes involucrados en la investigación. 


\section{Bibliografía}

1. Moreno G. La definición de salud de la Organización Mundial de la Salud y la interdisciplinariedad. Sapiens. Revista Universitaria de Investigación [Internet]. 2008 [citado 11 mayo 2019]. Disponible en: https://bit.ly/31RrNYm

2. Torres M, Ortiz R, Sigüencia W, Ortiz A, Añez R, Rojas J, Bermúdez V. Prevalencia de la obesidad en individuos adultos en las parroquias urbanas de la ciudad de Cuenca, Ecuador. Revista Síndrome cardiometabólico [Internet]. 2013 [citado 25 junio 2019]. Disponible en: http://132.248.9.34/hevila/Sindromecardiometabolico/2013/vol3/no4/1.pdf

3. Lucio R, Villacrés N, Henríquez R. Sistema de salud de Ecuador. Revista Salud pública de México [Internet]. 2011 [citado 18 mayo 2019]. Disponible en: http://www.scielo.org.mx/scielo. php?pid=S0036-36342011000800013\&script=sci_arttext

4. Medina FX, Aguilar A, Solé-Sedeño JM. Aspectos sociales y culturales sobre la obesidad: reflexiones necesarias desde la salud pública. Revista Nutrición clínica y dietética hospitalaria [Internet]. 2014 [citado 5 de mayo 2019]. Disponible en: https://revista.nutricion.org/PDF/ ASPECTOS-SOCIALES.pdf

5. Instituto Nacional de Estadística y Censos. República del Ecuador [Internet]. Nacimientos y Defunciones. Bases de Datos - Defunciones Generales. 2018 [citado 28 de abril 2019]. Disponible en: http://www.ecuadorencifras.gob.ec/ nacimientos-defunciones/

6. Le Breton M. On some combinatorial problems arising in the theory of voting games. Revista Mathematical Social Sciences [Internet]. 1990 [citado 13 de mayo 2019]. Disponible en: https://www.sciencedirect.com/science/article/ abs/pii/016548969090060K
7. Peñafiel A. Impacto del semáforo nutricional en los hábitos alimenticios de los estudiantes de la Universidad Politécnica Salesiana. Revista Research Journal [Internet]. 2016 [citado 14 de abril 2019]. Disponible en: https://dialnet.unirioja.es/servlet/articulo? codigo $=5920552$

8. Cahuasquí L, Flores E. El derecho a la alimentación en Ecuador desdeuna perspectiva de derechos humanos; 2011. Disponible en: http://repositorio.uasb.edu.ec/bitstream/10644/3652/1/ DH-Inf-2011-Cahuasqui-Flores-El\%20derecho.pdf

9. Calderón J, Alcívar C, Huacón A. Cómo incentivar a los ecuatorianos a tener una cultura alimenticia más saludable, para disminuir los índices de enfermedades que existen en la actualidad. Revista Caribeña de Ciencias Sociales [Internet]. 2015 [citado 24 de abril 2019]. Disponible en: https://ideas.repec.org/a/erv/ rccsrc/y2015i2015_0703.html

10. Cabezuelo G, Frontera, P. Enséñame a comer. Hábitos Pautas y Recetas para Evitar la Obesidad Infantil; 2007. Disponible en: http:// puentefichas.com/GMEL/FHGM/IINI/ extracto.pdf

11. Arizaga D. Análisis de la efectividad de los semáforos nutricionales como guía para el consumidor [Tesis de grado] Universidad de Cuenca; 2016. Disponible en: http://dspace.ucuenca. edu.ec/bitstream/123456789/25899/1/ TESIS.pdf

12. Coral S, Gómez C, Royo, M. Nutrición, salud y alimentos funcionales. Madrid: UNED, 2012. $124 \mathrm{p}$.

13. Universidad Laica Eloy Alfaro de Manabí. República del Ecuador [Internet]. Semaforización nutricional de los productos gastronómicos que se expenden en la playa Murciélago de la ciudad de Manta. S.f. [citado 28 de abril 2019]. Disponible en: https://bitlylink.com/HD4Wz 
14. De Luis Román D, Bellido D, García P. Dietoterapia, Nutrición Clínica y Metabolismo, Madrid: Díaz de Santos; 2012. Disponible en: https://bitlylink.com/X6bYR

15. Hoyos A, Yance K, Rendón A. Semaforizacion de productos de consumo, tendencia y culturizacion en los ecuatorianos. (U. Ecotec, Ed.) Observatorio de la Economía Latinoamericana; 2015. Disponible en: https://bitlylink.com/kNbk7

16. Instituto Ecuatoriano de Normalización. República del Ecuador [Internet]. Norma Técnica Ecuatoriana NTE INEN 1334-3:2011. Rotulado de productos alimenticios para consumo humano. Parte 3. Requisitos para declaraciones nutricionales y declaraciones saludables. Quito - Ecuador. s/f. 2018 [citado 3 de mayo 2019]. Disponible en: https://es.scribd.com/ document/287016805/1334-3-Norma-Tecnica-Ecuatoriana
17. Núńez S, Aulestiaz S, Borja E, Simancas D. Mortalidad por enfermedades isquémicas del corazón en Ecuador. 2001-2016: estudio de tendencias. Revista médica de Chile [Internet]. 2018 [citado 28 de mayo 2019]. Disponible en: https://bit. ly/2WkESXa

18. Moreno D, Santamaría D, Ludeña C, Barco A, Vásquez D, Santibáñez-Vásquez R. Enfermedad Cerebrovascular en el Ecuador: Análisis de los últimos 25 ańos de mortalidad, realidad actual y recomendaciones. Revista Ecuatoriana Neurología, [Internet]. 2016 [citado 12 de mayo 2019]. Disponible en: https://bit.ly/2Ar5H2n 